\title{
Subcutaneous fat necrosis associated with thrombocytosis in an infant following cardiac surgery
}

\author{
Catherine AE O'Blenes PhD, Patricia Berbari MD FRCSC, \\ Ronald M Zuker MD FRCSC FACS FAAP
}

CAE O'Blenes, P Berbari, RM Zuker. Subcutaneous fat necrosis associated with thrombocytosis in an infant following cardiac surgery. Can J Plast Surg 2002;10(1):37-40.

Subcutaneous fat necrosis in infancy is characterized by the appearance of subcutaneous nodules beneath a violaceous or erythematous plaque in infants exposed to ischemic stress from a variety of causes. It has been associated with hypercalcemia, hyperlipidemia and thrombocytopenia. This case of subcutaneous fat necrosis occurred 14 days after cardiac surgery in an infant taking enoxaparin who developed a paradoxical thrombocytosis. A potential etiological role for platelet sludging is discussed. Ultrasound images, not previously reported, showed thickened heterogeneous hypodense subcutaneous fat in the nodules and, thus, may be used to differentiate this condition from its surgical differential diagnoses.

Key Words: Subcutaneous fat necrosis; Thrombocytosis
Adiponécrose sous-cutanée associée à une thrombocytose chez un nourrisson après une intervention chirurgicale cardiaque

RÉSUMÉ : L'adiponécrose sous-cutanée se manifeste, chez les enfants soumis à un stress ischémique d'origine diverse, par la présence de nodules sous-cutanés sous des plaques violacées ou érythémateuses. Elle est associée à l'hypercalcémie, à l'hyperlipidémie et à la thrombocytopénie. L'adiponécrose est apparue, dans le présent cas, 14 jours après une intervention chirurgicale cardiaque chez un nourrisson prenant de l'énoxaparine et chez qui s'est installée une thrombocytose paradoxale. Suit une discussion sur le rôle étiologique possible de l'agglutination plaquettaire. L'échographie, dont on n'a pas fait mention antérieurement, a révélé la présence de matière grasse épaissie, hétérogène et hypodense sous-cutanée dans les nodules; l'image pourrait donc servir à distinguer l'affection de ses diagnostics différentiels chirurgicaux. 
Cubcutaneous fat necrosis (SFN) in newborns is a rare, Ousually benign disorder that most commonly presents during the first one to five weeks of life in full term infants affected by perinatal distress, and may occur later in infants who have undergone cardiac surgery $(1,2)$. It is characterized by the appearance of firm, mobile, subcutaneous nodules palpable beneath violaceous and/or erythematous plaques on the cheeks, shoulders, back, arms, hands and legs. Many etiological factors have been suggested, including maternal exposure to cocaine (3) or calcium channel blockers (4), gestational diabetes or pre-eclampsia (5), birth hypoxia $(6,7)$, local trauma $(6,7)$, anemia (8), cyanotic congenital heart disease (9), exogenous or elevated endogenous prostaglandins $(10,11)$ and hypothermia associated with cardiac surgery (2,12-15). A case of SFN associated with thrombocytosis, occurring after cardiac surgery under hypothermic conditions in an infant on subcutaneous enoxaparin is discussed. Ultrasound findings in the present case of SFN of the newborn are presented and, to the authors' knowledge, have not been reported previously. The potential etiological factors in this patient are discussed, and the eight other cases of SFN associated with congenital heart disease reported in the English literature are briefly examined.

\section{CASE PRESENTATION}

A female infant, with a birth weight of $3810 \mathrm{~g}$, was born at 41 weeks' gestation by cesarean section because of failure to progress, in the context of a prenatal ultrasound diagnosis of a complex congenital heart defect. The pregnancy had otherwise been unremarkable for the 31-year-old gravida 1 para 0 caucasian mother who was Rubella-immune, venereal disease research laboratory-negative, Rhesus antibodynegative, group B streptococcus- and human immunodeficiency virus-negative. Fetal echocardiography performed at term diagnosed transposition of the great arteries with a univentricular left ventricle-type heart, rudimentary right ventricle, ventricular septal defect and possible coarctation of the aorta. At birth, a thin meconium was noted. Apgar scores were nine at $1 \mathrm{~min}$ and 10 at $5 \mathrm{~min}$; however, the infant soon after birth became cyanosed with an oxygen saturation of $40 \%$. The infant was initially treated with 50\% oxygen. Prostaglandin E1 was then started to maintain patency of the ductus arteriosus and continued for five days before surgery, during which time oxygen saturations ranged between $85 \%$ to $90 \%$ on room air.

At five days of age, the infant underwent cardiac surgery, which consisted of atrial septostomy, reconstruction of the aortic arch with aortopulmonary connection, and a right modified Blalock-Taussig operation (shunt interposition between the subclavian artery and ipsilateral pulmonary artery). The operation was uneventful, performed under deep hypothermia with a cardiopulmonary bypass time of $120 \mathrm{~min}$, low flow time of $50 \mathrm{~min}$ and circulatory arrest time of $3 \mathrm{~min}$. This was the first of several surgeries required to create a Fontan circulation in this infant, in which the superior and inferior vena cava are connected to the pul-

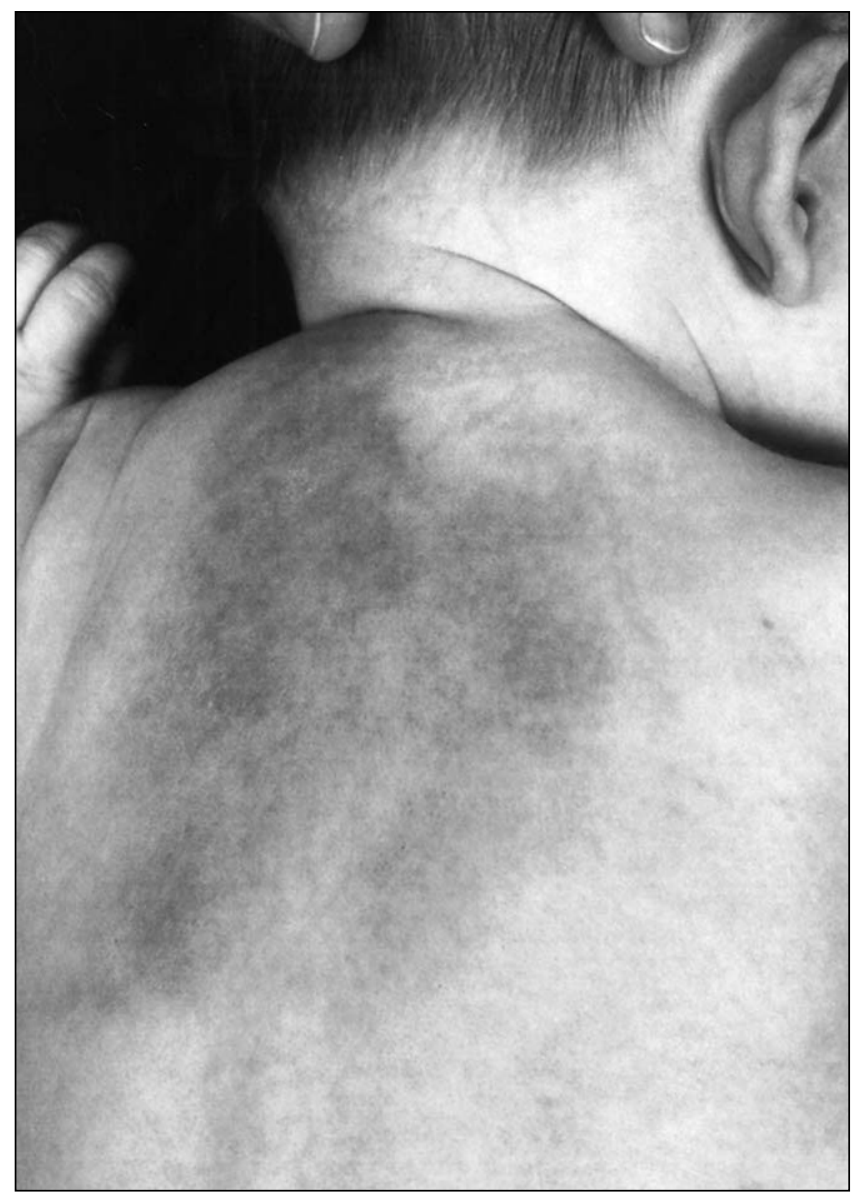

Figure 1) Violaceous indurated plaque with underlying subcutaneous nodules located on the back of the infant on postoperative day 16

monary artery to normalize the volume load to this univentricular heart. Postoperatively, prostaglandin E1 was discontinued and the infant was maintained on enoxaparin, spironolactone and hydrochlorothiazide.

The postoperative course was initially complicated by a superficial sternal wound infection on day 11 (age 16 days), which was treated with intravenous tobramycin and vancomycin.

On postoperative day 15 , the parents noted a violaceous and erythematous indurated plaque, approximately $12 \mathrm{~cm}$ in diameter, over the lower aspect of the neck and the upper back between the scapulae (Figure 1). Three firm, nontender, mobile, subcutaneous nodules measuring between 1 and $2 \mathrm{~cm}$ in diameter were palpable beneath the plaque. A diagnosis of SFN was made based on the characteristic clinical appearance of the lesion. An ultrasound study of the area was ordered to rule out other possible diagnoses such as lipoma, fibroma or hemangioma. The ultrasound showed increased thickness of the subcutaneous fat at the sites of the nodules and a heterogeneous hypodense appearance to the fat in the region corresponding to the nodules, with no evidence of calcification (Figure 2). Laboratory investigations revealed a normal serum calcium of $2.28 \mathrm{mmol} / \mathrm{L}$ (normal 2.25 to $2.75 \mathrm{mmol} / \mathrm{L}$ ) on postoperative day 16 . 


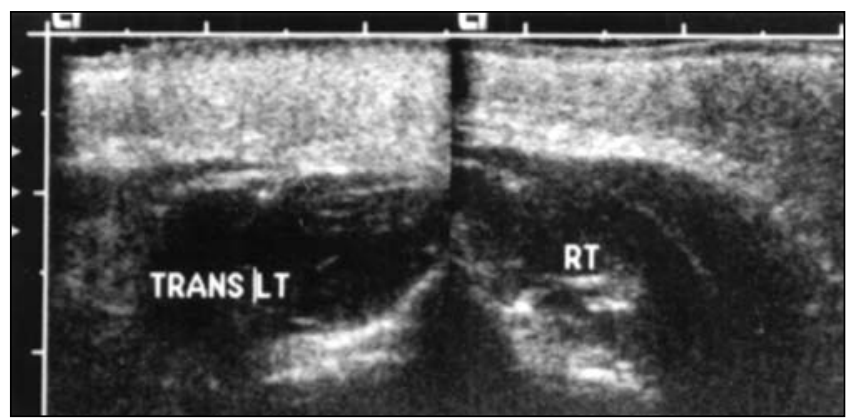

Figure 2) Ultrasound image of a subcutaneous nodule (left) compared with an area of normal subcutaneous fat (right) on the back of the infant on postoperative day 16, showing increased thickness of the subcutaneous fat and a heterogeneous hypodense appearance to the fat at the site of the nodule

Platelets were elevated on postoperative days 14 and 16 , respectively, at 746 and $857 \times 10^{9} / \mathrm{L}$ (normal 150 to $450 \times 10^{9} / \mathrm{L}$ ) and the white blood cell count was 17.8 and $21.1 \times 10^{9} / \mathrm{L}$ (normal 5 to $20 \times 10^{9} / \mathrm{L}$ ), while hemoglobin was normal. Partial thromboplastin time was $44.4 \mathrm{~s}$ (normal 25 to $60 \mathrm{~s}$ ), and the International Normalized Ratio was 1.04 on postoperative day 14 . The baby had otherwise done very well and was discharged home on enoxaparin, spironolactone and hydrochlorothiazide, with weekly calcium monitoring for hypercalcemia, which has previously been described in cases of SFN.

\section{DISCUSSION}

SFN in infancy is most commonly a benign condition in which the lesions are usually nontender and resolve spontaneously over one to three months (1). Although a diagnosis of SFN of infancy can be made on the basis of characteristic history and physical findings alone (Table 1), several important differential diagnoses should be considered, including sclerema neonatorum and hemangioma $(1,16)$. Sclerema neonatorum can be differentiated from SFN on the basis of history and physical examination. In contrast to SFN, which presents with discrete violaceous or red plaques, with underlying circumscribed nodules, sclerema neonatorum presents with the diffuse hardening of subcutaneous fat over the entire body except genitalia, soles and palms (7). Furthermore, sclerema neonatorum occurs in neonates who are severely ill with sepsis, congestive heart failure, pneumonia, intestinal obstruction, diarrhea or dehydration and is associated with a $75 \%$ mortality rate (7).

SFN has been associated with hypercalcemia in some patients. In a series of 11 patients with SFN diagnosed at The Hospital for Sick Children, Toronto, Ontario, between 1991 and 1998, 28\% developed hypercalcemia (1). Symptoms of hypercalcemia described in infants include failure to thrive, fever, vomiting, feeding difficulty, irritability, listlessness and seizures (7). If hypercalcemia occurs in association with SFN, it usually becomes apparent because the skin lesions resolve and may persist for as long as two months $(11,17)$. It is proposed that the hypercalcemia develops as a result of the release of calcium from calcifica-
TABLE 1

Clinical features of subcutaneous fat necrosis in infancy

\begin{tabular}{ll}
\hline & Subcutaneous fat necrosis in infancy \\
\hline Gestational age & $\begin{array}{l}\text { Term (most commonly), premature or } \\
\text { post-term }\end{array}$ \\
Labour & $\begin{array}{l}\text { Difficult labour common } \\
\text { with or without emergent cesarean section }\end{array}$
\end{tabular}

Associated

conditions Maternal factors

Exposure to cocaine

Exposure to calcium channel blockers

Gestational diabetes

Pre-eclampsia

Infant factors

Birth hypoxia

Local trauma

Anemia

Congenital heart disease

Prostaglandin E1

Prostaglandin E2

Cardiac surgery

Thrombocytopenia

Thrombocytosis

Onset One to six weeks (described in the literature at up to 20 months of age if following cardiac surgery)

Physical

examination Violaceous and/or erythematous plaques; subcutaneous, circumscribed, firm, mobile nodules located on trunk, face, arms, hands and legs

tions in necrotic fat and, perhaps more importantly, through increased 1,25-dihydroxy-vitamin $\mathrm{D}$ production by macrophages participating in the inflammatory process $(7,17)$. Hypercalcemia can be a life-threatening consequence of SFN and has been associated with a mortality rate of $15 \%$ (7). Thus, the management of this condition requires periodic monitoring for signs and symptoms of hypercalcemia, and serial calcium measurements. Burden and Krafchik (1) suggest monitoring serum calcium levels weekly for three to four months after the onset of SFN.

The precise etiology of SFN in the newborn has not been established; however, there are several proposed mechanisms. Birth trauma, hypoxia and hypothermia have all been implicated in the pathogenesis of SFN. Several authors have speculated that an underlying defect in lipid composition or metabolism may predispose certain individuals to fat necrosis when exposed to some form of ischemic stress $(2,8,10)$. In hypothermia, it is proposed that neonatal fat in some infants may undergo crystallization, leading to both direct adipocyte damage and ischemic stress through obstruction to arteriolar blood flow, resulting in fat necrosis (7). Infant fat contains an increased proportion of saturated 
fatty acids with a relatively high melting point compared with adult fat, which contains a greater proportion of unsaturated fatty acids with a lower melting point $(7,12)$. This may predispose infants to fat crystallization and necrosis secondary to hypothermia.

Interestingly, the areas affected by SFN appear to correspond with the localization of brown fat (10). Brown fat is believed to be the primary source of heat production in the newborn (9). Its high metabolic rate is further increased under hypothermic conditions, and this may make it particularly susceptible to necrosis under conditions of decreased tissue perfusion such as during cardiopulmonary bypass (9).

The present patient exhibited several potential etiological factors previously described in association with SFN, including perinatal distress, cyanotic congenital heart disease that required treatment with prostaglandin E1, hypothermic cardiac surgery and thrombocytosis.

To our knowledge, there have been eight reported cases of SFN associated with congenital heart disease in the English literature. In six of these cases, the patients underwent cardiac surgery with the use of cardiac bypass-induced generalized hypothermia and developed SFN one to four weeks after surgery. Consistent with these reports, the present patient developed SFN 15 days after surgery. Although the cyanosis associated with our patient's condition may have contributed to the development of SFN in this case, the time course suggests that hypothermic cardiac surgery played a central role. It seems likely that total ischemia time and decreased perfusion combined with hypothermia during cardiac surgery may contribute to the development of SFN in susceptible infants. Superficial cooling and generalized hypothermia induced by cardiac bypass are proposed to play roles in the initiation of SFN because the lesions are not limited to areas in contact with external cooling devices $(2,12)$.

In two of the reported cases of SFN associated with congenital heart disease, the patients did not undergo cardiac surgery but had been treated with prostaglandin E1 before the development of SFN $(10,11)$. The authors suggested a role for prostaglandins in the pathogenesis of SFN in susceptible infants $(10,11)$. Sharata et al $(11)$ describe the case of an infant with an Epstein anomaly of the tricuspid valve who required intravenous prostaglandin E1 to maintain a patent ductus arteriosus and improve oxygenation, and developed SFN after five days of treatment. Interestingly, once the therapy was stopped, no new nodules appeared, and the existing nodules resolved, although the patient continued to experience intermittent cyanosis with exertion (11). Raboi and Smith (10) describe the case of an infant with transposition of the great arteries who was treated with prostaglandin E1 for 22 days when brown fat necrosis was noted incidentally on a chest radiograph. A side effect of chronic prostaglandin E1 therapy is the hyalinization of peripheral arterioles and a decrease in lumen size, which could result in decreased perfusion and increased risk of fat necrosis (10). In contrast to the previously described patients, the present patient was treated with prostaglandin
E1 for five days before surgery (half-life 5 to $10 \mathrm{~min}$ ), but did not develop SFN until 15 days after surgery, suggesting that prostaglandin administration may not have played a significant causal role.

Thrombocytopenia has been described as a complication of SFN, normalizing with the resolution of the condition, and is thought to be due to sequestration of platelets at the areas of fat necrosis $(18,19)$. However, in our patient, an isolated thrombocytosis was detected one day before the detection of the lesions. It is uncertain whether this was a reactive thrombocytosis secondary to the SFN or whether it may have played a causal role through platelet sludging and decreased local perfusion to subcutaneous fat. Interestingly, the present patient was being treated with enoxaparin, which has been associated in a small number of instances with a secondary thrombocytosis $(20,21)$. There has been one other report (22), published in Italian, describing three cases of SFN in infants, all of which were associated with thrombocytosis. These were three term infants who required resuscitation at birth for severe perinatal hypoxia. In the first case, the infant developed an increased platelet count of $688 \times 10^{9} / \mathrm{L}$ on day 7 of life, and clinical evidence of SFN was apparent on day 10. In the second case, the infant exhibited SFN on day 4 of life and an elevated platelet count of $415 \times 10^{9} / \mathrm{L}$ was noted the following day. In the third case, an elevated platelet count of $656 \times 10^{9} / \mathrm{L}$ was noted on day 9, and clinical evidence of SFN did not become apparent until day 17. Our finding of increased platelets before the lesions becoming apparent is consistent with two of the above cases, suggesting a possible contributary role for thrombocytosis in the pathogenesis of SFN.

\section{CONCLUSIONS}

An infant with cyanotic congenital heart disease who developed subcutaneous fat necrosis likely secondary to decreased tissue perfusion and hypothermia during cardiac surgery was presented. Cases of subcutaneous fat necrosis have, until now, been published mainly in pediatric dermatology, radiology or histology journals. However, plastic surgery was the first service to be consulted in this case of SFN; hence, we were interested in reviewing this condition in the plastic surgery literature to facilitate its differentiation from its surgical differential diagnoses. SFN has a characteristic clinical appearance that may be confirmed by ultrasound findings, as presented in this case report, which have not, to the authors' knowledge, been reported elsewhere.

\section{REFERENCES}

1. Burden AD, Krafchik BR. Subcutaneous fat necrosis of the newborn: A review of 11 cases. Pediatr Dermatol 1999;16:384-7.

2. Chuang S-D, Chiu H-C, Chang C-C. Subcutaneous fat necrosis of the newborn complicating hypothermic cardiac surgery. Br J Dermatol 1995;132:805-10.

3. Carraccio C, Papadimitriou J, Feinberg P. Subcutaneous fat necrosis of the newborn: link to maternal use of cocaine during pregnancy. Clin Pediatr 1994;33:317-8.

4. Rosbotham JL, Johnson A, Haque KN, Holden CA. Painful subcutaneous fat necrosis of the newborn associated with intra-partum use of a calcium channel blocker. Clin Exp Dermatol 1998;23:19-21. 
5. Lusk RP, Greiman MC. Subcutaneous fat necrosis in infancy. Otolaryngol Head Neck Surg 1988;99:520-3.

6. Cook JS, Stone MS, Hansen JR. Hypercalcemia in association with subcutaneous fat necrosis of the newborn: studies of calciumregulating hormones. Pediatrics 1992;90:93-6.

7. Hicks MJ, Levy ML, Alexander J, Flaitz CM. Subcutaneous fat necrosis of the newborn and hypercalcemia: Case report and review of the literature. Pediatr Dermatol 1993;10:271-6.

8. Varan B, Gurakan B, Ozbek N, Emir S. Subcutaneous fat necrosis of the newborn associated with anemia. Pediatr Dermatol 1999;16:381-3.

9. Stephenson TJ, Variend S. Visceral brown fat necrosis in postperinatal mortality. J Clin Pathol 1987;40:896-900.

10. Raboi CA, Smith W. Brown fat necrosis in the setting of congenital heart disease and prostaglandin E1 use: a case report. Pediatr Radiol 1999;29:61-3.

11. Sharata H, Postellon DC, Hashimoto K. Subcutaneous fat necrosis, hypercalcemia, and prostaglandin E. Pediatr Dermatol 1995;12:43-7.

12. Silverman AK, Michels EH, Rasmussen JE. Subcutaneous fat necrosis in an infant, occurring after hypothermic cardiac surgery. J Am Acad Dermatol 1986;15:331-6.

13. Glover MT, Catterall MD, Atherton DJ. Subcutaneous fat necrosis in two infants after hypothermic cardiac surgery. Pediatr Dermatol 1991;8:210-2.

14. Blake HA, Goyette EM, Lyter CS, Swan H. Subcutaneous fat necrosis complicating hypothermia. J Pediatr 1955;46:78-80.
15. Collins HA, Stahlman M, Scott HW. The occurrence of subcutaneous fat necrosis in an infant following induced hypothermia used as an adjuvant in cardiac surgery. Ann Surg $1953 ; 138: 880-5$

16. Anderson DR, Narla LD, Dunn NL. Subcutaneous fat necrosis of the newborn. Pediatr Radiol 1999;29:794-6.

17. Finne PH, Sanderud J, Aksnes L, Bratlid D, Aarskog D. Hypercalcemia with increased and upregulated 1,25-dihydroxy vitamin $\mathrm{D}$ production in a neonate with subcutaneous fat necrosis. J Pediatr 1988;112:792-4.

18. Lewis A, Cowen P, Rodda C, Dyall-Smith D. Subcutaneous fat necrosis of the newborn complicated by hypercalcemia and thrombocytopenia. Australas J Dermatol 1992;33:141-4.

19. Wolach B, Raas-Rothschild A, Vogel R, Choc L, Metzker A Subcutaneous fat necrosis with thrombocytopenia in a newborn infant. Dermatologica 1990;181:54-5.

20. Williams E. Thrombocytosis associated with low-molecular-weightheparin. Ann Intern Med 1997;126:742-3.

21. Ziaja K, Simka M, Krupowies A, Dugaj M, Ludyga T. Thrombocytosis after prophylactic administration of enoxaparin: unexpected findings in a Polish prospective multicenter trial on the efficacy and safety of enoxaparin in the prevention of postoperative thromboembolism. Int Angiol 1999;18:65-9.

22. Turba F, Bianchi C, Cella D, Rondanini GF. [Thrombocytosis and neonatal subcutaneous adiponecrosis]. Minerva Pediatr 1994;46:343-6. 